\title{
A Cluster-based Routing Algorithm for WSN based on Residual Energy of the Nodes
}

\author{
Supriya Das \\ Department of Computer Science \\ Pondicherry Central University, Pondicherry, India
}

\author{
P. Shanthi Bala \\ Department of Computer Science \\ Pondicherry Central University, Pondicherry, India
}

\begin{abstract}
Wireless sensor networks can be said as the latest development or improvement in the field of wireless communication. However the advantages of the WSN become limited due to the limited resources, especially low-capacity battery of the sensor nodes. Thus, energy aware routing is an important issue for prolonging the lifetime of the WSN. Among all the basic routing protocols based on the network structure, clustering algorithms have been the much researched upon because of their scalable and energy efficient nature. In this paper, a clustering routing algorithm has been proposed to increase the sensor network lifetime by choosing the cluster heads based on their residual energy and the distance between the cluster members and corresponding cluster heads.
\end{abstract}

\section{Keywords}

Wireless sensor networks, LEACH, clustering, remaining energy, routing algorithm, EECS.

\section{INTRODUCTION}

Wireless sensor networks (WSN) is one of the latest advancements in the field of wireless communication networks that have become part and parcel of today's world, be it in the military fields or in the mere household appliances, used to gather data from the area of interests like the hostile, barren terrain or the human body itself. However the major limitation faced by the WSN is the low battery life of the sensor nodes, though researches are going on to increase the lifetime of the batteries keeping in mind the miniature size of the sensor nodes. But, till then to maximize the lifespan of the WSN, the routing protocols can be designed accordingly that can reduce the overall data flow and energy consumption in the network.

Though the sensor networks are based on the wireless communication schemes, the demands of the WSN cannot be satisfied by the protocols designed for other traditional wireless networks like ad-hoc or cellular networks. This is because of the characteristics of the WSN like- application specificity, huge number and large density of the nodes, lack of global identification scheme etc. Many routing protocols have been proposed for the WSN till date among which the hierarchical routing algorithms have been voted for as the best ones because of the scalable, energy efficient nature, its capacity of reducing data latency and enhancing the lifetime of the network.

In this paper, a hierarchical algorithm is proposed that uses residual energy and the distance between the cluster heads and the sink as the parameters to improve the efficiency of the algorithm. Section 2 gives an insight into the two algorithms, LEACH that was first algorithm, developed to bring out the goodness of the clustering phenomenon and EECS, the first algorithm to consider the energy of the individual nodes while forming the clusters. Section 3 describes the proposed algorithm followed by the simulation and its result analysis in the section 4 . Section 5 concludes the paper with a brief note about the scope of future work in the proposed phenomenon.

\section{RELATED WORK}

This section includes the description of those two algorithms that were amongst the first algorithms to exploit the hierarchical nature and energy while developing the algorithm, LEACH and EECS respectively. The proposed work has been compared against these two algorithms, as the protocol is also based on the clustering scheme and energy as one of its main parameters.

LEACH [1]: Proposed as an application-specific, energy efficient, randomized clustering protocol. LEACH was actually defined for reducing the amount of redundant data from flowing in the network, thereby saving energy. LEACH was defined assuming the following characteristics: random selection of cluster heads from amongst the randomly self-configured clusters, localized control and co-ordination amongst the clusters, reduction of the communication overload by compressing the data locally, low energy media access control, applicationspecific protocol. However there certain problems with the traditional LEACH algorithm, such as:

- The clusters are randomly selected which may give rise to uneven distribution of energy throughout the network.

- While selecting the cluster head, neither its residual energy nor its distance from the other nodes or base station is considered.

- It assumes homogenous nodes and uniform energy consumption of the $\mathrm{CHs}$

- LEACH uses single-hop transmission to communicate with the base station, so LEACH can't be used for a large scale network.

- Leach assumes that the sensor nodes always have some data to send.

LEACH introduced the concept of rounds in hierarchical algorithms. The LEACH algorithm progresses in rounds and each round is divided into two stages; the first stage being the setting up of the clusters and the cluster heads, so is known as the set up phase and the second stage which handles the actual data transmission from the cluster members to the cluster heads and then to the base 
station/base stations and is called the steady-state phase. In the set-up phase, the nodes are chosen to be the cluster heads on the basis of the random number generated by them which lies between 0 and 1 and then compared against the threshold value $T_{t h r}$

$$
T_{\text {thr }}= \begin{cases}\frac{p}{1-p\{r \bmod (1 / p)\}} & \begin{array}{l}
n \in G \\
\text { else }
\end{array}\end{cases}
$$

EECS [4]: Proposed to better suit the periodic data gathering applications of WSN. Data gathering is a basic yet very critical process in many applications of WSNs where hierarchical mechanism is used. Energy efficient clustering schema (EECS) is similar to LEACH clustering scheme, where the network is partitioned into a group of clusters with one cluster head in each cluster and the communication between cluster head and BS is direct. However, cluster formation mechanism is different in the sense that, in LEACH, the cluster formation is based on the minimum distance of nodes to their corresponding cluster head whereas, in EECS, automatic sizing of the clusters occurs on the basis of cluster distance from the base station. The EECS was developed with the following primary goals to be satisfied:

- Fully distributed manner,

- Low-control overhead,

- Load balanced clustering phenomenon

But still it is not without its disadvantages. In large scale networks, this algorithm considering direct communication of the cluster head with the sink is not feasible. Also the intra-cluster processes are not emphasized in this algorithm.

\section{PROPOSED WORK}

Before describing the proposed algorithm let us go through some of the assumptions considered for the wireless sensor network scenario. The network model for the proposed algorithm assumes the following points:

i) The nodes in the network are static.

ii) Nodes are location-aware, which can be known using GPS

iii) Each node need to access only the information of its neighbors in one hop.

iv) All nodes are homogeneous having same processing, communication and initial energy.

v) The transmission ranges of nodes are adjustable.

vi) The sink is stationary.

The energy model is assumed as a simple model for the radio hardware energy consumption where the transmitter dissipates energy to run the radio electronics and the power amplifier, and the receiver dissipates energy to run the radio electronics. Both the free space $\left(\mathrm{d}^{2}\right.$ power loss) and the multipath fading ( $\mathrm{d}^{4}$ power loss) channel models are used in this paper, depending on the distance between the transmitter and receiver. Power control can be used to invert this loss by appropriately setting the power amplifier, if the distance is less than a threshold, the free space model is used; otherwise, the multipath model is used. Thus, to transmit a $k$ bit message a distance $d$, the radio expends

$$
E_{T x}(k, d)=\left\{\begin{array}{l}
k E_{\text {elec }}+k \varepsilon_{f s} d^{2} \ldots . . d<d_{0} \\
k E_{\text {elec }}+k \varepsilon_{m p} d^{4} \ldots . d \geq d_{0}
\end{array}\right.
$$

and to receive this message, the radio expends:

$$
E_{R x}(k)=k E_{\text {elec }}
$$

where, $E_{\text {elec }}$ is the electronics energy. $\epsilon_{f s}$ and $\epsilon_{m p}$ are the amplifier energy of the free space model and the multipath model. $d_{0}$ is the minimum distance for deciding the parameter value, given by $d_{0}=\sqrt{\frac{\varepsilon_{f s}}{\varepsilon_{m p}}}$

\subsection{Proposed algorithm}

1. To calculate the average residual energy of each sensor in the network.

$$
F_{e}=\sum_{j \in N_{i}} \frac{E_{j \text { current }}}{\left|N_{i}\right| \cdot E_{i \text { current }}}
$$

Where, $\mathrm{N}_{\mathrm{i}}$ is the set of neighbors, which are in radio range of node $\mathrm{i},\left|\mathrm{N}_{\mathrm{i}}\right|$ is the total number of nodes in the neighbor list and $E_{i \text { current }}$ is the current residual energy of node $i$.

2. To calculate the average distance

$$
F_{d}=\sum_{j \in N_{i}} \frac{d_{i, j}^{2}}{\left|N_{i}\right|}
$$

3. To calculate the threshold value

$$
T_{t h r}=\alpha_{1} F_{e}+\alpha_{2} F_{d}
$$

Where, $\alpha_{1}$ and $\alpha_{2}$ are the weights of the factors and $\alpha_{1}<\alpha_{2}\left(\alpha_{1}+\alpha_{2=1}\right)$. i.e. in the proposed algorithm more importance given to average residual energy of a node than the distance.

First of all the neighbors of each node is detected, by calculating the distance amongst the nodes. When nodes have sufficient information about its neighbors, such as distance and current energy, nodes broadcast its $T_{\text {thr }}$ value to neighbors. According to $\mathrm{T}_{\mathrm{thr}}$, each node selects the candidate node which has the minimal $\mathrm{T}_{\text {thr }}$, and sends elect $_{\text {msg }}$ to the candidate node. The nodes which receive the most elect $_{\mathrm{msg}}$ in neighbors, will announce that the cluster-head nodes are elected, and all non-cluster-head nodes chose one nearest cluster-head to join the cluster.

4. The optimal number of clusters $K$ can be expressed as follow:

$$
k=\sqrt{\frac{N}{2 \pi}} \frac{D \sqrt{\varepsilon_{f s}}}{\sqrt{E_{\text {elec }}(N+1)+\varepsilon_{m p} l^{4}}}
$$

Where, $D$ is the length of the side of network area and $l$ is the distance from $\mathrm{CH}$ to sink node. 
The round time $T_{\text {current }}$ is defined at the beginning of the round $\mathrm{R}_{\text {current }}$. It depends on the optimal cluster size, rather than using a constant round time $\mathrm{T}$ for every round in the network life. Then one can define the current round time $\mathrm{T}_{\text {current }}$ as follows:

$$
T_{\text {current }}=N F_{\text {avg. }}\left(M_{\text {min }} * \sigma+\lambda\right)
$$

where, $\mathrm{NF}_{\mathrm{avg}}$ is the average number of frames for a cluster with size $1 / \mathrm{k}$. $\left(\mathrm{M}_{\min }{ }^{*} \sigma+\lambda\right)$ is the frame time of a cluster which has the minimum size (the minimum number of nodes $\mathbf{M}_{\min }$ ).After defining the round time $\mathrm{T}_{\text {current }}$ for the current round, the base station sends the cluster information and the modified $\mathrm{T}_{\text {current }}$ to all nodes in the network, therefore each node can determine its cluster and its time slot in the TDMA schedule.

\section{SIMULATION AND RESULT ANALYSIS}

WSN routing protocols can be simulated with a variety of tools like MATLAB, OMNET++, OPNET, NS2 etc. In this paper, the network simulation tool NS2 has been used to run the proposed algorithm against two of the basic algorithms, LEACH and EECS because of the hierarchical and energy efficient characteristics respectively.

Here, their performances are compared based on the rate of the deaths of the nodes and their energy consumption in the same network scenario.

A. Simulation scenario: The basic assumption made about the simulation parameters are as mentioned next. 200 nodes have been randomly distributed over the sensing area of size 300x300 m and sink node having the location co-ordinates $(95,250)$. Each node is provided with the initial energy of $3 \mathrm{~J}$. The energy consumed in the electronics circuit of each node to transmit or receive a signal, $E_{\text {elec }}$ is $100 \mathrm{~nJ} /$ bit. Also for each node, the energy consumed by the amplifier to transmit at a short distance and a longer distance is $\mathrm{E}_{\mathrm{fs}}=20 \mathrm{pJ} / \mathrm{bit} / \mathrm{m}^{2}$ and $E_{m p}=0.0015 \mathrm{pJ} / \mathrm{bit} / \mathrm{m}^{4} . \quad \mathrm{d}_{0}$ is taken as $100 \mathrm{~m}$ and the transmitted data is $3000 \mathrm{bits}$

B. Simulation analysis: In this paper, it has been chosen to compare the performances of the algorithms with the proposed algorithm by considering parameters like network lifetime and energy consumption. Here, the metrics like first node dead (FND), half of the nodes dead (HND) and last node dead (LND) have been selected to describe the network lifetime. Less the number of nodes die, higher is the lifetime of the network.

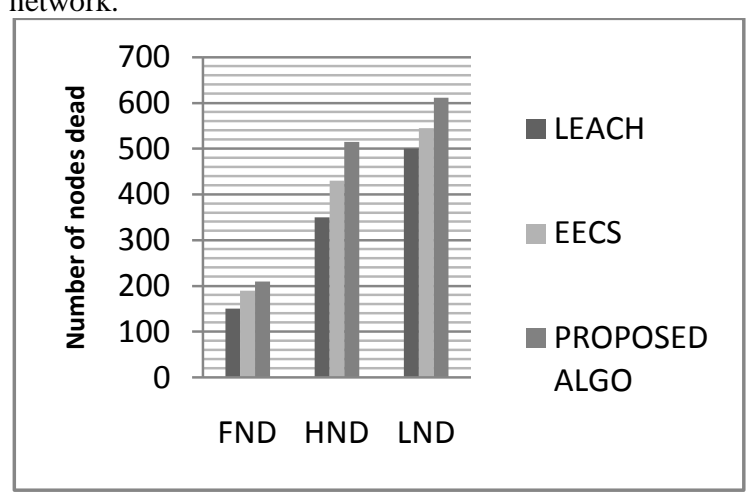

Fig.1: Comparison of the network lifetime among the algorithms
In figure 1 , the $\mathrm{x}$-coordinate depicts the number of rounds run and $y$-coordinate denote the number of nodes dead per round. The above figure shows that in comparison with the LEACH AND EECS, the proposed algorithm is more effective by the percentage as shown in the table 1 below:

Table 1: Efficiency of the proposed algorithm over LEACH \& EECS

\begin{tabular}{|c|c|c|}
\hline & LEACH & EECS \\
\hline FND & $40 \%$ & $11 \%$ \\
\hline HND & $47 \%$ & $20 \%$ \\
\hline LND & $23 \%$ & $12 \%$ \\
\hline
\end{tabular}

Next, the figure 2 below indicates total energy consumption at different time periods (no. of rounds). In the simulation each node have $3 \mathrm{~J}$ initial energy and number of nodes are 300, thus total energy of the network is $3 * 300=900 \mathrm{~J}$. As one can see from the figure above, all the nodes consumed their energy and died after 495 rounds, when routing algorithm is LEACH. In EECS all nodes exhausted their energy after 550 rounds and as seen, the nodes in the proposed algorithm are able to survive up to 660 rounds (average) hence proving superiority of proposed algorithm over leach and EECS as $33 \%$ and $16 \%$ enhanced network life time respectively.

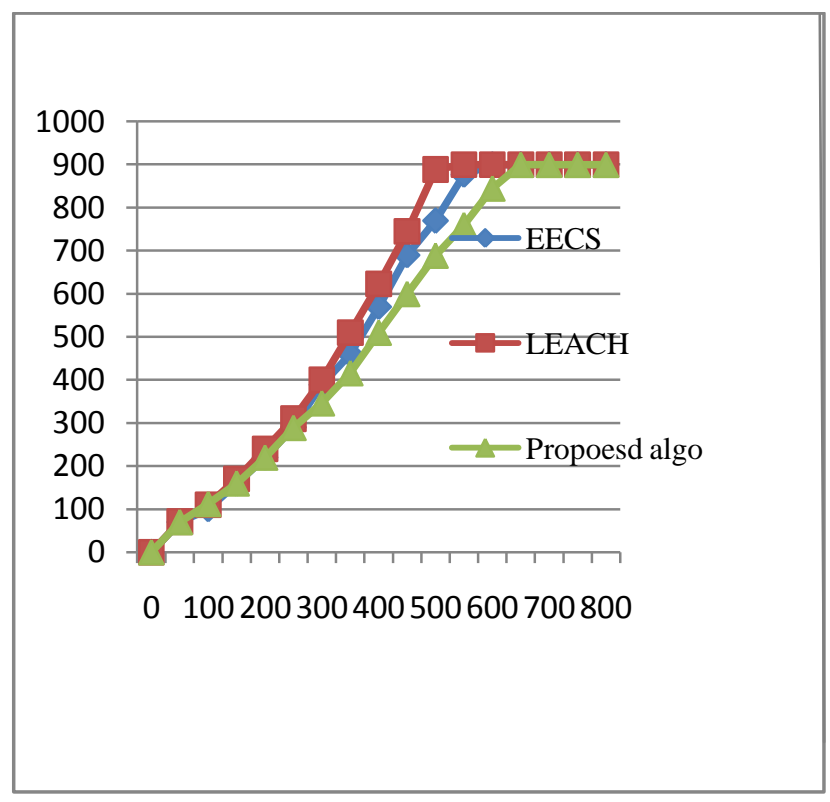

Fig.2: Energy consumption of the algorithms in different rounds 


\section{CONCLUSION \& FUTURE WORKS}

In this paper, a cluster-based protocol for wireless sensor networks has been proposed. This algorithm is designed to enhance the overall lifetime of the network by increasing the lifetime of each of the nodes and for this the residual energy of the nodes and the distance between the nodes and their corresponding cluster-heads and the same between the cluster heads and the sink are considered. Also incorporated, is the self-selection of the optimal number of clusters here unlike the static number of clusters calculated at the beginning of LEACH based on the number of nodes. Simulation software NS2 has been used to simulate the aspects like nodes alive, network lifetime, energy consumption. Comparison has shown the proposed algorithm to be effectively better than its counterparts.

As a part of the future works, one can simulate the above algorithms in networks of different sizes and measure their performances, energy consumption based on the different location the sink node and in different orders of the network span.

\section{REFERENCES}

[1] W. Heinzelman, A. Chandrakasan, and $\mathrm{H}$. Balakrishnan, "An application-specific protocol architecture for wireless microsensor networks," IEEE Transaction on Wireless Communications, 2002, vol. 1, no. 4, pp. 660-670.

[2] Performance Comparison of LEACH and LEACHC Protocols by NS2

[3] O. Younis and S. Fahmy, "HEED: A Hybrid, Energy-Efficient, Distributed Clustering Approach for Ad Hoc Sensor Networks," IEEE Transactions on Mobile Computing, vol. 3, no. 4, pp. 366-379, 2004.

[4] M. Ye, C. Li, G. Chen, and J. Wu, EECS: An Energy Efficient Clustering Scheme in Wireless Sensor Networks, National Laboratory of Novel Softaware Technology,Nanjing University, China.

[5] D. H. Nam, "An efficient ad-hoc routing using a hybrid clustering method in a wireless sensor network," in Wireless and Mobile Computing, Networking and Communications, 2007. WiMOB 2007. Third IEEE International Conference on, 2007, pp. 60-60.

[6] S. Bandyopadhyay, E. Coyle, An energy efficient hierarchical clustering algorithm for wireless sensor networks, in: Proceedings of the 22nd Annual Joint Conference of the IEEE Computer and Communications Societies (INFOCOM 2003), San Francisco, California, April 2003.

[7] W. B. Heinzelman. "Application-Specific Protocol Architectures for Wireless Networks". PhD thesis, Massachusetts Institute of Technology, June 2000.

[8] A. Depedri, A. Zanella and R. Verdone, "An Energy Efficient Protocol for Wireless Sensor Networks" In Proc. AINS, 2003, pp. 1-6.

[9] L. Lijun, W. Hunt, and C. Peng, "Discuss in a round rotation policy of hierarchical route in wireless sensor networks," in proceedings IEEE International Conference WiCOM, 2006, pp. 1-5.
[10] X. Fan and Y. Song, "Improvement on leach protocol of wireless sensor network," in In Proceedings of the International Conference on Sensor Technologies and Applications, 2007, pp. 260-264.

[11] Rajashree.V.Biradar, Dr.S.R. Sawant, Dr. R. R. Mudholkar, Dr. V.C .Patil "Multihop Routing In Self-Organizing Wireless Sensor Networks" IJCSI International Journal of Computer Science Issues, Vol. 8, Issue 1, January 2011.

[12] An Energy Efficient Clustering Algorithm Based on Residual Energy and Concentration Degree in Wireless Sensor Networks (Yuzhong Chen, and Yiping Chen), Proceedings of the Second Symposium International Computer Science and Computational Technology(ISCSCT '09), Huangshan, P. R. China, 26-28,Dec. 2009, pp. 306309

[13] Zhu Yong, Qing Pei, A Energy-Efficient Clustering Routing Algorithm Based on Distance and Residual Energy for Wireless Sensor Networks, 2012 International Workshop on Information and Electronics Engineering (IWIEE)

[14] EEHC: Energy efficient heterogeneous clustered scheme for wireless sensor networks (Dilip Kumar a,*, Trilok C. Aseri b,1, R.B. Patel c,2), Computer Communications 32 (2009) 662-667

[15] M. J. Handy, M. Haase, D. Timmermann, "Low Energy Adaptive Clustering Hierarchy with Deterministic Cluster-Head Selection," Proceedings of the 4th IEEE Conference on Mobile and Wireless Communications Networks, Stockholm, Sweden, pp. 368-372, Sept. 2002.

[16] Bian, X.X.; Liu, X.C.; Cho, H. Study on a ClusterChain Routing Protocol in Wireless Sensor Networks. In Proceedings of the 3rd International Conference on Communications and Networking

[17] Tao, Y.; Zheng, Y.L. The Combination of the Optimal Number of Cluster-Heads and Energy Adaptive Cluster-Head Selection Algorithm in Wireless Sensor Networks. In Proceedings of International Conference on Wireless Communications, Networking and Mobile Computing

[18] Zhang, H.Z.; Chen, P.P.; Gong, S.L. Weighted Spanning Tree Clustering Routing Algorithm Based on Leach. In Proceedings of the 2nd International Conference on Future Computer and Communication (ICFCC 2010), Wuhan, China, 2124 May 2010; pp. V2-223-V222-227.

[19] Jang, K.Y.; Kim, K.T.; Youn, H.Y. An Energy Efficient Routing Scheme for Wireless Sensor Networks. In Proceedings of the International Conference on Computational Science and its Applications (ICCSA 2007), Kuala Lumpur, Malaysia, 26-29 August 2007; pp. 399-404.

[20] Soroush Naeimi,Hamidreza Ghafghazi, Chee-Onn Chow, Hiroshi Ishii. "A survey on the taxonomy for Cluster-based Routing Protocols for homogeneous wireless sensor networks". ISSN 1424-8220. 